\title{
Sensory gating in primary insomnia
}

\author{
Ilana S. Hairston, ${ }^{1,2}$ Lisa S. Talbot, ${ }^{2}$ Polina Eidelman, ${ }^{2}$ June Gruber ${ }^{2,3}$ and Allison G. Harvey ${ }^{2}$ \\ ${ }^{1}$ Psychiatry Department, Addiction Research Center, University of Michigan, Rachel Upjohn Bldg, 4250 Plymouth Rd, Ann Arbor, \\ MI 48109 5740, USA \\ ${ }^{2}$ Psychology Department, University of California, Berkeley, CA, USA \\ ${ }^{3}$ Psychology Department, Yale University, New Haven, CT, USA
}

Keywords: event-related potentials, human, K-complex, sensory processing, spindles

\begin{abstract}
Although previous research indicates that sleep architecture is largely intact in primary insomnia (PI), the spectral content of the sleeping electroencephalographic trace and measures of brain metabolism suggest that individuals with PI are physiologically more aroused than good sleepers. Such observations imply that individuals with PI may not experience the full deactivation of sensory and cognitive processing, resulting in reduced filtering of external sensory information during sleep. To test this hypothesis, gating of sensory information during sleep was tested in participants with primary insomnia $(n=18)$ and good sleepers $(n=20)$. Sensory gating was operationally defined as (i) the difference in magnitude of evoked response potentials elicited by pairs of clicks presented during Wake and Stage II sleep, and (ii) the number of K complexes evoked by the same auditory stimulus. During wake the groups did not differ in magnitude of sensory gating. During sleep, sensory gating of the N350 component was attenuated and completely diminished in participants with insomnia. P450, which occurred only during sleep, was strongly gated in good sleepers, and less so in participants with insomnia. Additionally, participants with insomnia showed no stimulus-related increase in K complexes. Thus, PI is potentially associated with impaired capacity to filter out external sensory information, especially during sleep. The potential of using stimulus-evoked $\mathrm{K}$ complexes as a biomarker for primary insomnia is discussed.
\end{abstract}

\section{Introduction}

Primary insomnia (PI) is the persistent difficulty initiating or maintaining sleep, or experiencing non-restorative sleep, with subjective daytime impairment in the absence of another sleep-related, physical or psychiatric condition (American Academy of Sleep Medicine, 2005). Recent studies report important differences between the sleep of individuals with PI and good sleepers, including elevated beta (Lamarche \& Ogilvie, 1997; Perlis et al., 2001a) and gamma (Perlis et al., 2001b; Krystal et al., 2002) power in the electroencephalographic (EEG) trace during non-rapid eye movement (NREM) sleep, and elevated brain metabolism during sleep (Nofzinger et al., 2006; Buysse et al., 2008).

It has been proposed that PI is the outcome of a confluence of behavioural, cognitive and physiological events leading to central nervous system hyperarousal (Edinger et al., 2001), which counters inhibitory processes essential for entering and maintaining sleep (Spielman et al., 1987; Perlis et al., 1997; Espie, 2002). Thus, during sleep and especially sleep onset, there is a failure to fully deactivate sensory processing, thereby interfering with sleep initiation and maintenance (Bonnet \& Arand, 1997; Perlis et al., 1997; Espie, 2002; Harvey, 2002; Turcotte \& Bastien, 2009).

Correspondence: Dr I. S. Hairston, ${ }^{1}$ Psychiatry Department, as above.

E-mail: ilanahai@med.umich.edu

Received 3 December 2009, revised 16 March 2010, accepted 19 March 2010
One prediction derived from this conceptualization is that individuals with PI would be impaired in their ability to filter out external stimuli during sleep. Several markers for reduced or inhibited sensory processing during sleep have been described, including an eventrelated potential (ERP) model of pre-attentive processes and stimulusevoked K-complexes (KCs). For example, using the auditory sensory gating paradigm, Kisley et al. (2001) reported release from gating of N100 during NREM sleep in healthy participants. Auditory sensory gating is a commonly used physiological procedure that assesses inhibitory mechanisms and sensory gating (Patterson et al., 2008). It is assumed that sensory gating reflects pre-attentional habituation to a repetitive stimulus (Adler et al., 1998), thus filtering out redundant information.

Stimulus-evoked KCs may also reflect gating of sensory information. KCs are discrete slow waves typical of Stage II sleep, which can be evoked by interference such as a loud noise or respiratory obstruction. It has been proposed that $\mathrm{KCs}$ have a sleep protective function (Bastien et al., 2000; Nicholas et al., 2002; Colrain, 2005).

Despite a significant amount of work on sensory processing during sleep, few studies have assessed this phenomenon in PI. Recently, two studies administered a mismatch negativity paradigm, a measure of involuntary attention, in participants with PI. Both studies demonstrated that at sleep onset participants with PI had sustained mismatch negativity (Yang \& Lo, 2007; Bastien et al., 2008), supporting the notion that cognitive processes are not inhibited by sleep in PI. 
The present study aimed to expand on these findings. Using the sensory gating ERP paradigm we aim to assess the ability of PI participants to exclude sensory information during sleep. We hypothesize that individuals with PI will show sustained gating during sleep. Additionally, we hypothesize that PI will be associated with reduced capacity to recruit sleep protective mechanisms, resulting in less evoked KCs in response to stimulus presentation.

\section{Methods}

All procedures were approved by the University of California, Berkeley, Committee for the Protection of Human Subjects, and conforming to The Code of Ethics of the World Medical Association (Declaration of Helsinki). Written informed consent was obtained from each participant prior to the beginning of the study.

\section{Participants}

Twenty primary insomnia (INS) and twenty good sleepers (GS) were recruited via ads posted on the Internet. Two INS participants were subsequently excluded due to disclosure of the use of sleep aid medications. Participants were screened for medical history, current health status and subjective sleep quality using a 7-day sleep diary and the Duke Structured Interview Schedule for DSM-IV-TR and International Classification of Sleep Disorders, second edition (Edinger et al., 2000, 2004), and for Axis I disorders using the Structured Clinical Interview for DSM-IV-TR Axis I Disorders, Research Version, Non-Patient Edition (SCID-I/NP, Michael et al., 2002). GS were excluded for any history of Axis I and sleep disorders. Insomnia participants were excluded for other current Axis I disorders, current use of sleep aid medications, and if in the week prior to assessment they did not meet research criteria for insomnia (i.e. at least three of the 7 days in which sleep onset latency was $=30 \mathrm{~min}$ or awake after sleep onset was $=30 \mathrm{~min}$; Edinger et al., 2004). Finally, participants from either group were excluded for evidence of occult sleep disorders with a respiratory distress index $(\mathrm{RDI})>5$, and/or periodic limb movement $(\mathrm{PLM})$ index $>15$, based on polysomnographic (PSG) recordings.

\section{Environment and instruments}

The study was conducted at the Sleep and Psychological Disorders Laboratory at the University of California, Berkeley. PSG data were acquired by a Compumedics Siesta802 Wireless system, at a sampling rate of $256 \mathrm{~Hz}$, using PSG Online. EEG, electrocardiography (ECG) and electrooculography (EOG) data were filtered at $0.3-48 \mathrm{~Hz}$; electromyography (EMG) data were filtered at $10-100 \mathrm{~Hz}$. A $60-\mathrm{Hz}$ notch filter was applied to remove electrical artefacts. Data were acquired and stored on a Dell Optiplex GX280, Intel Pentium ${ }^{\circledR}$, running Windows XP. PSG data were visually staged, off-line, according to standard criteria (Rechtschaffen \& Kales, 1968), using Compumedics' Profusion PSG2+.

The ERP stimulus consisted of pairs of 50- to 72-dB white-noise clicks, each of $10 \mathrm{~ms}$, generated by Audacity (Audacity Developer Team, http://audacity.sourceforge.net/), presented $500 \mathrm{~ms}$ apart, $\pm 50 \mathrm{~ms}$ jitter, with an inter-trial interval of $3000 \mathrm{~ms}$. Stimuli were presented with Psyscope (http://psy.ck.sissa.it/) run on an Apple iBook, and delivered via E2c Sound Isolating Earphones (Shure Incorporated, Niles IL; info@shure.com). On each stimulus delivery a trigger mark was sent to the PSG amplifier recording event onset simultaneously with PSG acquisition.

\section{Procedures}

For 7 days prior to initial assessment, participants completed a daily self-report sleep diary, immediately upon awakening. The diary was used to determine subjective sleep onset latency (sSOL), subjective wake after sleep onset (sWASO), subjective number of awakenings (sNumW) and subjective total sleep time (sTST). In addition, sleepiness was assessed twice daily, upon awakening and at bedtime using the Stanford Sleepiness Scale (SSS; Hoddes et al., 1973) also included in the diaries. A similar sleep diary, without the SSS, was administered on subsequent overnight visits in the laboratory.

The first visit to the laboratory occurred during the day, at which time participants' diaries were reviewed, followed by an interview with a trained researcher to assess Axis I and sleep disorders. PI participants were included only if they met research criteria for insomnia according to their sleep diaries the previous week (i.e. $30 \mathrm{~min}$ of SOL and/or WASO for at least three of the 7 days; Edinger et al., 2004), and if they met criteria for primary insomnia according to the Duke Structured Interview Schedule for DSM-IV-TR and International Classification of Sleep Disorders, second edition (Edinger et al., 2000). Additionally, participants completed self-report questionnaires assessing demographic characteristics, severity of insomnia symptoms using the Insomnia Severity Index (ISI, scale 1-5; Bastien et al., 2001), symptoms of depression using the Beck Depression Inventory (BDI-II; Beck et al., 1996) and anxiety measures using Spielberger's State-Trait Anxiety Inventory (STAI; Spielberger et al., 1970).

Participants slept in the lab for two, non-consecutive, nights 5-14 days apart. The first night was used to confirm the absence of occult sleep disorders. Participants were asked to arrive at the lab about $1 \mathrm{~h}$ before their habitual bedtime. After preparing for bed, participants were fitted with a standard PSG set-up, including C3, C4, O1, O2, A1 and A2 EEG electrodes, two ocular electrodes (EOG) referenced to A1 and A2, and two submental EMG electrodes. Additionally, participants' heart rate, blood oxygen, nasal and oral airflow, thoracic and abdominal effort, and leg motion were monitored.

On the second (ERP) night, participants were asked to arrive at the laboratory $2 \mathrm{~h}$ before their habitual bedtime. $\mathrm{Cz}$ was added to the standard PSG montage to record ERPs, and ECG and thoracic effort were monitored. After PSG setup, participants were informed that they would be watching a film without sound, with subtitles only, for $1 \mathrm{~h}$ while an auditory stimulus was presented, and were instructed to ignore the sounds and focus on the film. To keep sound intensity constant for both sleep and wake, participants were instructed to select a sound intensity they felt would least interrupt their sleep. The minimum allowed was $50 \mathrm{~dB}$, and the maximum used was $72 \mathrm{~dB}$, by adjusting the volume on the computer. Sound intensity was determined later by connecting the headphones to a sound pressure meter. Individual ratings were not recorded.

Waking ERP consisted of five blocks of 11 min (170 trials) each, with a 2- to 5-min break between blocks. Stimulus presentation started $5 \mathrm{~min}$ after the beginning of the film. At the end of five blocks, participants went to bed. During the night, blocks of $11 \mathrm{~min}$ were presented every 40-60 min, starting $1 \mathrm{~h}$ after sleep onset and ending about $1 \mathrm{~h}$ before scheduled wake up time. Each participant received $6-$ 8 blocks during the night. On both mornings, participants completed sleep diaries.

\section{Data processing}

Scoring of sleep stages was completed off-line by trained research assistants, who manually scored each 30 -s epoch according to the 
criteria of Rechtschaffen \& Kales (1968). Subsequently, artefact-free raw data from $\mathrm{Cz}, \mathrm{A} 1, \mathrm{~A} 2, \mathrm{EMG}, \mathrm{ECG}$, and left and right EOG were imported into Matlab (http://www.mathworks.com). This conversion step resulted in the loss of absolute voltage information, and all ERP data are reported in relative $Z$-scores, with the mean set to zero.

For ERP analysis, two blocks acquired during Stage II sleep in the latter half of the night were used. This strategy was employed to reduce the effects of homeostatic pressure on EEG observed in the early part of the night, and because the stimulus presentation in other sleep stages often resulted in transition into Stage II (Fig. 1).

EEGLAB Toolbox, version 6.1 (Delorme \& Makeig, 2004), was used to visualize and process ERP data. Independent Component Analysis (ICA) was used to remove eye blinks and heartbeat artefacts. EEG data were then filtered with a high-pass filter of $1 \mathrm{~Hz}$ and lowpass filter of $45 \mathrm{~Hz}$, and $\mathrm{Cz}$ was re-referenced to the average of A1 and A2. ERP responses were averaged, for each click (S1, S2) separately, each trial consisting of a window of $650 \mathrm{~ms}(100 \mathrm{~ms}$ before, $550 \mathrm{~ms}$ after, stimulus onset). Prior to averaging, blocks were again visually inspected and trials with large motion artefacts were removed.

Magnitude and latency values for the different components of the ERP waveform were detected using a filter designed to identify peak values and their relative latency for each waveform. To assess gating, the ratio of $\mathrm{S} 2 / \mathrm{S} 1$ was calculated for each component, such that a ratio close to 0 indicated robust suppression while a ratio of 1 or greater indicated no sensory gating (Kisley et al., 2001).

For evoked KC analysis, all blocks during Stage II or mixed Stage II/III throughout the night were used. KCs were visually identified using the criteria of Rechtschaffen \& Kales (1968). Additionally, to maintain inter-rater reliability, raters adhered to the following criteria: an event was considered a $\mathrm{KC}$ if it included a negative followed by a $100-\mu \mathrm{V}$ or greater positive deflection, with a duration $0.5-1 \mathrm{~s}$.
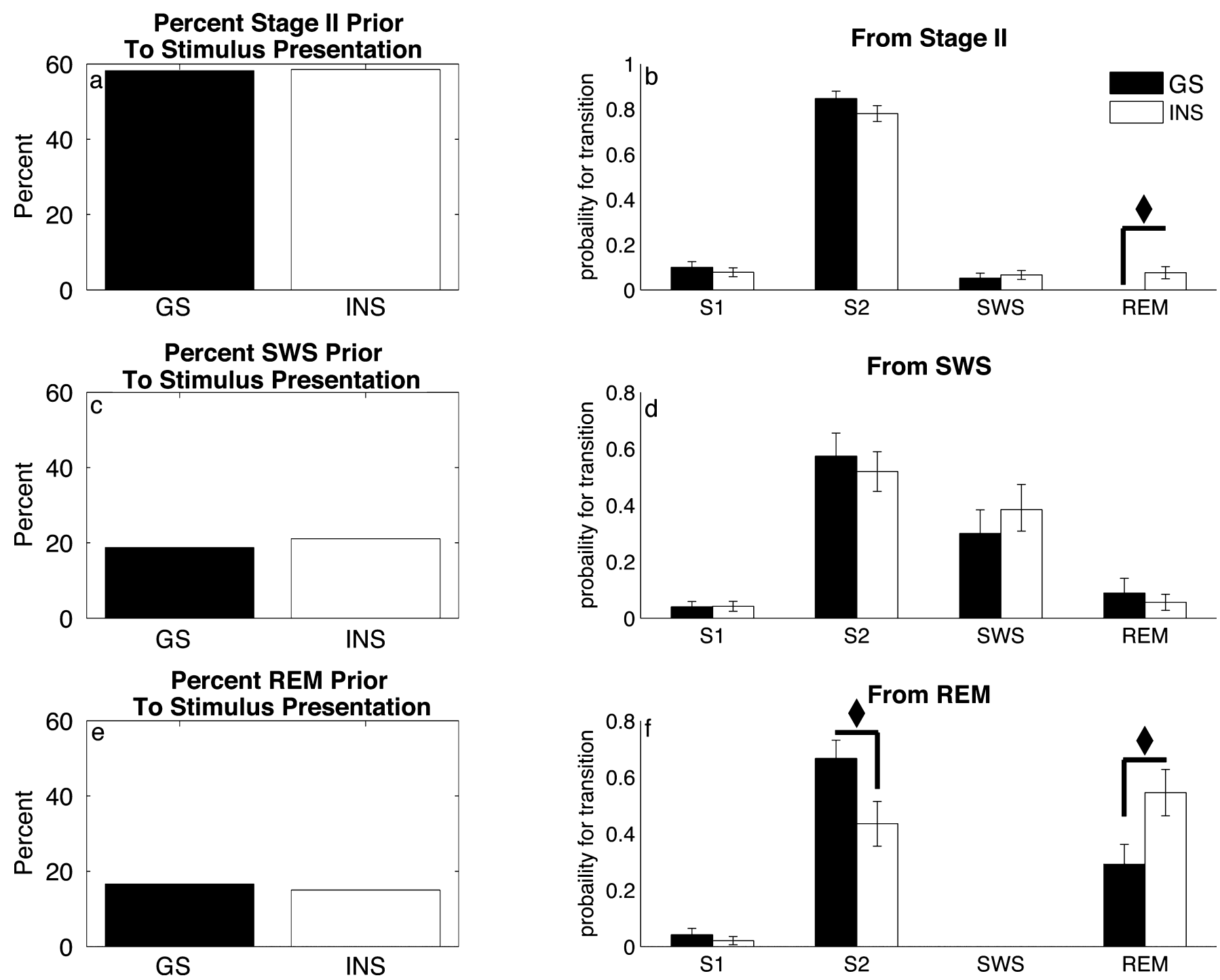

FIG. 1. The effects of stimulus presentation on sleep stage. The 11 min prior to each block of stimulus presentation was used as baseline, wherein the predominant sleep stage was defined as the stage that accounted for $>50 \%$ of this time period. Panels a, c and e demonstrate the percentage of baseline epochs in Stages II (a), SWS (c) and REM (d), respectively. No differences between groups were found. Transition probability was defined as the distribution of sleep stages in terms of proportion of the 11 min during stimulus presentation spent in Stages I, II, SWS and REM (b, d, and f). (b) When baseline was predominantly Stage II, participants tended to remain in Stage II $(P<0.001)$, with a higher probability of transition into REM in the INS group $(P=0.012)$. From SWS (d) participants tended to transition to Stage II or remain in SWS $(P<0.001)$, with no group differences. From REM (f) participants tended to transition to Stage II or remain in REM $(P<0.001)$, with a higher probability for INS to remain in REM compared with GS $(P=0.003)$. ' $\checkmark$ ' indicate a difference between groups. 
Furthermore, the $\mathrm{KC}$ had to be observed in all central channels (i.e. $\mathrm{Cz}, \mathrm{C} 3, \mathrm{C} 4)$. The number of KCs per epoch of Stage II and mixed Stage II/III epochs during stimulus presentation was compared with the number of $\mathrm{KCs}$ observed in the equivalent amount of time (11 min) immediately preceding each block. The operational definition of evoked $\mathrm{KCs}$ in this study was the ratio of the number of KCs during stimulus presentation to the number of KCs per epoch observed in the equivalent amount of time (11 $\mathrm{min})$ immediately preceding each block. As Stage II sleep is defined by the occurrence of two distinct markers, KCs and spindles, a similar quantification was performed on spindles to assess whether the effects of stimulus presentation were specific to the mechanism involved in KC. Spindles were identified as a $12-$ to $16-\mathrm{Hz}$ modulation, of $0.5-1.5$ day, that tapered at the beginning and end of the event.

\section{Statistical analysis}

Chi-squared, $t$-tests and Mann-Whitney Wilcoxon $U$-test were used to compare the groups on demographic and psychological variables. $t$-tests and MANOVAS were used for sleep variables, and analysis of group differences in the magnitude of ERP components. One-sample $t$-tests were used to determine if the gating ratio (S2/S1) was significantly $<1$ (= no gating). Where appropriate, partial eta squared $\left(\eta_{\mathrm{p}}{ }^{2}\right)$ was reported as an approximation of effect size.

\section{Results}

Table 1 summarizes demographics, sleep and mood of participants. In both groups, two-thirds were women $\left(\chi_{3}^{2}=0.114, P=1.0\right)$, with similar mean $\left(t_{39}=1.515, P=0.138\right)$ and range (GS, 18-57; INS, 20-56) of age. INS participants were more likely to be employed $\left(\chi_{3}^{2}=13.13, P=0.022\right)$ and had a marginally higher income $\left(\chi_{3}^{2}=6.96, P=0.073\right)$. Mann-Whitney $U$-tests were used to compare groups on the ISI, BDI-II and STAI. The INS group scored higher than GS on the ISI $(Z=-4.13, P<0.001)$, BDI $(Z=-3.60, P<0.001)$, and on both state and trait anxiety subscales of the STAI (state anxiety: $Z=-3.06, P=0.002$; trait anxiety: $Z=-3.37, P=0.001$ ).

According to the weeklong sleep diaries, INS had longer SSOL and more sWASO compared with GS participants (Table 2). Statistical analysis was not performed on these measures as they were used as exclusionary criteria for the INS group. However, the INS group reported nearly three-fold the number of awakenings (sNumW, $t$-test ${ }_{39}=-4.17, P<0.001, \eta_{\mathrm{p}}{ }^{2}=0.37$ ) with no difference in sTST $\left(t\right.$-test $\left._{39}=-0.67, P=0.51\right)$. Only 14 INS and ten GS completed the SSS reliably, twice a day, for the whole week. A MANOVA with time of day and group as the within-subject factors on the SSS yielded a significant group difference in the morning $\left(F_{1,23}=12.1, P=0.037\right.$, $\left.\eta_{\mathrm{p}}{ }^{2}=0.18\right)$ but not the evening $\left(F_{1,23}=0.24, P=\mathrm{NS}\right)$, due to higher sleepiness ratings in the morning in the INS group.

To assess whether sleeping in the lab and the ERP manipulation affected subjective sleep quality the average sSOL, sWASO and sNumW from the sleep diaries completed at home was used to calculate percentage change on these measures during the nights in the laboratory. Repeated-measures ANOVAS with night (Acclimation, ERP) as within-subject variables, and group (INS, GS) as the fixed variable were run on the percentage change value of each parameter. As can be seen in Table 3, GS had a larger increase on all subjective measures on both nights (sSOL: $F_{1,38}=5.44, \quad P=0.027$, $\eta_{\mathrm{p}}{ }^{2}=0.16$; sWASO: $F_{1,38}=10.72, P=0.003, \eta_{\mathrm{p}}{ }^{2}=0.28$; sNumW: $\left.F_{1,38}=19.02, P=0.001, \eta_{\mathrm{p}}^{2}=41\right)$, without interactions with night (all $F<1$ ). One-sample $t$-tests were performed on each measure,
TABLE 1. Demographic, sleep and mood measures of participants collected on their first visit to the laboratory

\begin{tabular}{|c|c|c|c|}
\hline & $\begin{array}{l}\text { Insomnia } \\
(\mathrm{INS}, n=18)\end{array}$ & $\begin{array}{l}\text { Good sleepers } \\
(\mathrm{GS}, n=20)\end{array}$ & Significance \\
\hline \multicolumn{4}{|l|}{ Age (years) } \\
\hline Mean $\pm \mathrm{SD}$ & $30.95 \pm 11.5$ & $36.65 \pm 12.2$ & \multirow{2}{*}{$t<1.0$} \\
\hline Range & $18-56$ & $18-57$ & \\
\hline Sex (female/male) & $14 / 7$ & $14 / 6$ & NS \\
\hline \multicolumn{4}{|l|}{ Ethnicity } \\
\hline African American & 2 & 1 & \multirow[t]{5}{*}{$\mathrm{NS}$} \\
\hline Asian/Pacific Islands & 7 & 3 & \\
\hline Hispanic & 0 & 1 & \\
\hline White & 10 & 12 & \\
\hline Other & 1 & 3 & \\
\hline Education (median) & 2 years college & 4 years college & NS \\
\hline Income $(\$$, range $)$ & $25-50,000$ & $50-75,000$ & $P=0.073$ \\
\hline \multicolumn{4}{|l|}{ Employment } \\
\hline Full time & 9 & 11 & \multirow[t]{4}{*}{$P=0.022$} \\
\hline Part time & 9 & 5 & \\
\hline Retired & 1 & 0 & \\
\hline Unemployed & 1 & 4 & \\
\hline \multicolumn{4}{|l|}{ Marital status } \\
\hline Single & 17 & 17 & \multirow[t]{2}{*}{ NS } \\
\hline Married/life partner & 3 & 3 & \\
\hline \multicolumn{4}{|l|}{ ISI } \\
\hline Median & 17 & 3.5 & \multirow[t]{2}{*}{$P<0.001$} \\
\hline Range & $5-26$ & $1-12$ & \\
\hline \multicolumn{4}{|l|}{ BDI-II } \\
\hline Median & 8 & 0 & \multirow[t]{2}{*}{$P<0.001$} \\
\hline Range & $0-18$ & $0-5$ & \\
\hline \multicolumn{4}{|l|}{ STAI state } \\
\hline Median & 39 & 30 & \multirow[t]{2}{*}{$P=0.002$} \\
\hline Range & $26-58$ & $25-41$ & \\
\hline \multicolumn{4}{|l|}{ STAI trait } \\
\hline Median & 44 & 32 & \multirow[t]{2}{*}{$P<0.001$} \\
\hline Range & $30-56$ & $25-45$ & \\
\hline
\end{tabular}

The INS group were more likely to be employed, and had slightly higher education. The INS group scored higher on the insomnia severity index (ISI), Beck Depression Inventory (BDI) and State and Trait Anxiety Indices (STAI).

TABLE 2. Results of 1-week sleep diary information collected prior to first visit to the laboratory

\begin{tabular}{llll}
\hline Parameter & Insomnia (INS) & Good sleepers $(G S)$ & $P$-value \\
\hline Diary information & $(n=18)$ & $(n=20)$ & \\
$\quad$ sSOL & $36.4 \pm 26.2$ & $8.3 \pm 8.7$ & $\mathrm{~N} / \mathrm{A}^{*}$ \\
sWASO & $33.9 \pm 28.8$ & $10.4 \pm 20.4$ & $\mathrm{~N} / \mathrm{A}^{*}$ \\
sTST & $430.9 \pm 56.7$ & $443.2 \pm 44.9$ & 0.510 \\
sNumW & $2.2 \pm 1.4$ & $0.7 \pm 0.5$ & 0.001 \\
SSS & $(n=14)$ & $(n=10)$ & \\
$\quad$ Morning & $3.25 \pm 3.0$ & $2.56 \pm 2.2$ & 0.001 \\
$\quad$ Evening & $3.69 \pm 4.5$ & $3.91 \pm 3.8$ & 0.101 \\
\hline
\end{tabular}

*The subjective sleep onset latency (sSOL) and subjective wake after sleep onset (sWASO) were not included in statistical analyses as they were inclusion criteria for the INS group. The groups did not differ in subjective total sleep time (sTST) but INS had significantly more subjective awakenings (sNumW). INS participants scored higher than controls on the Stanford sleepiness scale (SSS) in the mornings, but not evenings.

comparing the percentage change with a predicted mean of $100 \%$, for each group separately. GS had significant increases in sWASO and sNumW (all $P<0.05$ ), and close to significant increase in sSOL 
TABLE 3. Mean percentage change in SSOL, sWASO and sNumW in the lab compared with sleeping at home

\begin{tabular}{|c|c|c|c|c|c|c|}
\hline & \multicolumn{3}{|c|}{ Insomnia (INS) $(n=18)$} & \multicolumn{3}{|c|}{ Good sleepers (GS) $(n=20)$} \\
\hline & $\begin{array}{l}\text { Change (\%) } \\
(99 \% \mathrm{CI})\end{array}$ & $t$-value & $P$-value & $\begin{array}{l}\text { Change (\%) } \\
(99 \% \mathrm{CI})\end{array}$ & $t$-value & $P$-value \\
\hline \multicolumn{7}{|l|}{ Acclimation } \\
\hline sSOL & 181 & 1.37 & 0.191 & 484 & 1.97 & 0.068 \\
\hline sWASO & 274 & 1.69 & 0.113 & 654 & 2.90 & 0.012 \\
\hline sNumW & 148 & 1.30 & 0.215 & 519 & 2.96 & 0.010 \\
\hline sSOL & 62 & -4.61 & 0.001 & 384 & 1.91 & 0.076 \\
\hline sWASO & 125 & 0.82 & 0.426 & 757 & 2.91 & 0.011 \\
\hline sNumW & 143 & 1.55 & 0.142 & 522 & 4.43 & $>0.001$ \\
\hline
\end{tabular}

GS had significant increases on both nights in sWASO and sNumW, while INS showed a decline in sSOL on event-related potential (ERP) night. sSOL, subjective sleep onset latency; sWASO, subjective wake after sleep onset; sNumW, subjective awakenings.

TABLE 4. Summary of polysomnography analysis

\begin{tabular}{|c|c|c|c|c|c|}
\hline & \multicolumn{2}{|c|}{ Insomnia (INS) $(n=18)$} & \multicolumn{2}{|c|}{ Good sleepers (GS) $(n=20)$} & \multirow[b]{2}{*}{$P$-value } \\
\hline & Acclimation & ERP night & Acclimation & ERP night & \\
\hline oWASO & $40.98 \pm 48.2$ & $27.2 \pm 27.0$ & $19.75 \pm 22.6$ & $18.00 \pm 28.7$ & $0.047^{*}$ \\
\hline SE & $83.01 \pm 13.1$ & $84.51 \pm 12.7$ & $90.99 \pm 7.4$ & $90.81 \pm 8.4$ & $0.013 *$ \\
\hline \multicolumn{6}{|l|}{ Sleep } \\
\hline Stage II (\%) & $54.04 \pm 9.3$ & $57.51 \pm 9.9$ & $56.33 \pm 6.6$ & $55.81 \pm 7.8$ & NS \\
\hline SWS (\%) & $16.43 \pm 7.6$ & $15.73 \pm 8.4$ & $17.45 \pm 7.4$ & $19.85 \pm 9.0$ & NS \\
\hline REM (\%) & $20.13 \pm 6.5$ & $18.97 \pm 6.3$ & $18.72 \pm 6.8$ & $17.31 \pm 7.3$ & NS \\
\hline Arousals $/ \mathrm{h}$ & $11.35 \pm 6.3$ & $9.73 \pm 4.2$ & $13.28 \pm 7.0$ & $11.38 \pm .6 .8$ & NS \\
\hline
\end{tabular}

Presentation of the auditory stimulus during the ERP night had no effect on any of the sleep parameters. The two groups differed in their sleep onset latency (oSOL), wake after sleep onset (oWASO) and sleep efficiency (SE, $P=0.042,0.047$ and 0.013 , respectively), but not in the distribution of sleep stages and number of arousals. *Significant main effect of group; ${ }^{\dagger}$ significant main effect of night. REM, rapid eye movement sleep; SWS, slow-wave sleep; oTST, objective total sleep time.

(acclimation: $P=0.068$; ERP: $P=0.076$ ). INS only showed a significant decrease in $\operatorname{SSOL}(P=0.001)$.

PSG data analysis was largely consistent with participants' self reports (Table 4). Overall objective SOL (oSOL) was significantly greater in INS than in controls $\left(F_{1,41}=4.39, P=0.042, \eta_{\mathrm{p}}{ }^{2}=0.10\right)$ as was oWASO $\left(F_{1,41}=4.15, P=0.047, \eta_{\mathrm{p}}{ }^{2}=0.09\right)$. Sleep efficiency (SE), defined as the percentage time in bed spent asleep, was lower in INS $\left(F_{1,41}=6.71, P=0.013, \eta_{\mathrm{p}}{ }^{2}=0.14\right)$. The average oSOL was reduced on the ERP night in the INS group, but no interaction of night by group was found (all $F<1.0$ ).

To determine the effects of the ERP manipulation on sleep quality we assessed whether stimulus presentation caused a change in sleep stage. For each 11-min block, the 11 min prior to stimulus onset was used as a pre-stimulus baseline. Sleep stage of baseline was determined as the prevalent stage in $51 \%$ or more of the epochs during this time. Transition probability was defined as the distribution of sleep stages during stimulus presentation from each baseline period. Figure 1 depicts the results of this analysis. There were no baseline periods with $>50 \%$ Stage I; Stage II (Fig. 1a) accounted for about two-thirds of the baseline blocks, the remainder being equally distributed between slow-wave sleep (SWS) and REM (Fig. 1c and e, respectively), with no group differences $(F<1.0)$.

For each baseline sleep stage, multivariate ANOVAS were performed with sleep stage during stimulus presentation as the dependent factors, and group as the fixed factor. To adjust for multiple comparisons the error term was set to 0.005 . When the stimulus was introduced during Stage II there was a main effect of stage $\left(F_{4,123}=165.74, P<0.001\right.$, $\left.\eta_{\mathrm{p}}{ }^{2}=0.80\right)$ as both groups tended to remain in Stage II during stimulus presentation. There was no effect of group $(F<1.0)$, but there was a significant interaction of group with sleep stage $\left(F_{1,125}=6.70, P=0.011, \eta_{\mathrm{p}}{ }^{2}=0.50\right)$, as INS were more likely to transition to REM $(P=0.09)$ compared with GS $(P=0.0$; post hoc $F=6.68, P=0.012, \eta_{\mathrm{p}}{ }^{2}=0.05$, Fig. $\left.1 \mathrm{~b}\right)$. When the stimulus was introduced during SWS there was a main effect of stage $\left(F_{4,123}=110.66, P<0.001, \eta_{\mathrm{p}}{ }^{2}=0.87\right)$, as both groups tended to transition into Stage II or remain in SWS during stimulus presentation, compared with REM or Stage I; there was no statistical difference between SWS and Stage II $(P=0.346)$. There was no effect of group and no interaction of group with stage $(F<1.0$, Fig. 1d). When the stimulus was introduced during REM sleep there was a main effect of stage $\left(F_{4,123}=38.09, P<0.001, \eta_{\mathrm{p}}{ }^{2}=0.51\right)$ as both groups tended to transition into Stage II during stimulus presentation or stay in REM, compared with SWS or Stage I; there was no difference between REM and Stage II $(P=0.139)$. There was no effect of group $(F<1.0)$, but there was a significant interaction of group with sleep stage $\left(F_{1,125}=4.88, P=0.003, \eta_{\mathrm{p}}{ }^{2}=0.12\right)$, as INS were more likely to remain in REM $(P=0.58)$ compared with GS $(P=0.33$; post hoc $\left.F=5.15, P=0.029, \eta_{\mathrm{p}}{ }^{2}=0.13\right)$, and less likely to transition to Stage 
II $(P=0.40)$ compared with GS $(P=0.64 ;$ post hoc $F=4.81$, $\left.P=0.035, \eta_{\mathrm{p}}{ }^{2}=0.12\right)$.

Figure 2 depicts the group and state effects on ERP. During wake (Fig. 2a and c) the evoked response consisted of two major components, a positive deflection with a latency of around $300 \mathrm{~ms}$ (P300) and a negative deflection around $350 \mathrm{~ms}$ (N350). During sleep (Fig. 1b and d) P300 was diminished, but a delayed equivalent of N350 and a late positive component around $450 \mathrm{~ms}$ (P450) appeared. Using a repeated-measures ANOVA, with stimulus order (S1, S2) as a within-subject variable, the magnitude of P300 was significantly reduced for S2 in both groups $\left(F_{1,38}=47.41, P<0.001, \eta_{\mathrm{p}}{ }^{2}=0.60\right)$, with a main effect of group $\left(F_{1,38}=7.82, P=0.009 \eta_{\mathrm{p}}{ }^{2}=0.20\right)$, due to lower amplitude observed in the INS group, with no interaction $\left(F_{1,38}=1.56 P=0.220\right)$. Repeated-measures ANOVA with stimulus order and arousal state (Wake, Stage II sleep) as the within-subject variables on the N350 component yielded a significant difference between the magnitude of $\mathrm{S} 1$ and $\mathrm{S} 2$ across groups $\left(F_{1,35}=41.52\right.$, $\left.P<0.001, \eta_{\mathrm{p}}{ }^{2}=0.55\right)$, a main effect of group $\left(F_{1,35}=6.22\right.$, $\left.P=0.018, \eta_{\mathrm{p}}{ }^{2}=0.16\right)$ due to reduced magnitude of N350 in the INS group, mainly during wake, and an interaction of stimulus order with group $\left(F_{1,35}=4.71, P=0.037, \eta_{\mathrm{p}}{ }^{2}=0.12\right)$ due to greater group differences in $\mathrm{S} 1\left(M_{\mathrm{diff}}=1.72, P=0.008\right)$ than $\mathrm{S} 2\left(M_{\mathrm{diff}}=0.93\right.$,
$P=0.077)$. No effect of arousal state $\left(F_{1,35}=1.27, P=0.270\right)$, and no interactions with state (all $F<1.0$ ) were found. Repeated-measures ANOVA with stimulus order as a within-subject variable was performed on P450 during sleep, and yielded a main effect of stimulus order $\left(F_{1,35}=8.15, P=0.007, \eta_{\mathrm{p}}{ }^{2}=0.18\right)$, no main effect of group $\left(F_{1,35}=1.43, P=0.239\right)$, but a significant interaction of group with stimulus order $\left(F_{1,35}=6.37, P=0.016, \eta_{\mathrm{p}}{ }^{2}=0.15\right)$, due to significant difference between S1 and S2 for GS $\left(M_{\text {diff }}=3.58 P<0.001\right)$ but not INS $\left(M_{\mathrm{diff}}=0.22 P=0.822\right)$. Similar analyses performed on the latencies for each component yielded no significant effects (all $F<1.0$, data not shown).

The results of gating ratio (GR) analyses are depicted in Fig. 3. Univariate ANOVA on P300 GR yielded no group difference $\left(F_{1,35}=0.19, P=0.669\right)$, and for both groups the ratio was significantly $<1$ (one-sample $t$-test, INS: $t_{17}=-6.93, P<0.001$; GS: $\left.t_{18}=-6.41, P<0.001\right)$. A repeated-measure ANOVA on N350 GR, with arousal state as the within-subject variable and group as the fixed variable, yielded a main effect of arousal state $\left(F_{1,36}=4.89\right.$, $\left.P=0.034, \eta_{\mathrm{p}}{ }^{2}=0.12\right)$, with no effect of group $\left(F_{1,36}=1.19\right.$, $P=0.283)$ and no interaction $\left(F_{1,36}=1.75, P=0.195\right)$. One-sample $t$-tests revealed that during wake GR was statistically $<1$ for both groups (INS: $t_{17}=-10.93, P<0.001$; GS: $t_{19}=-7.18, P<0.001$ ),
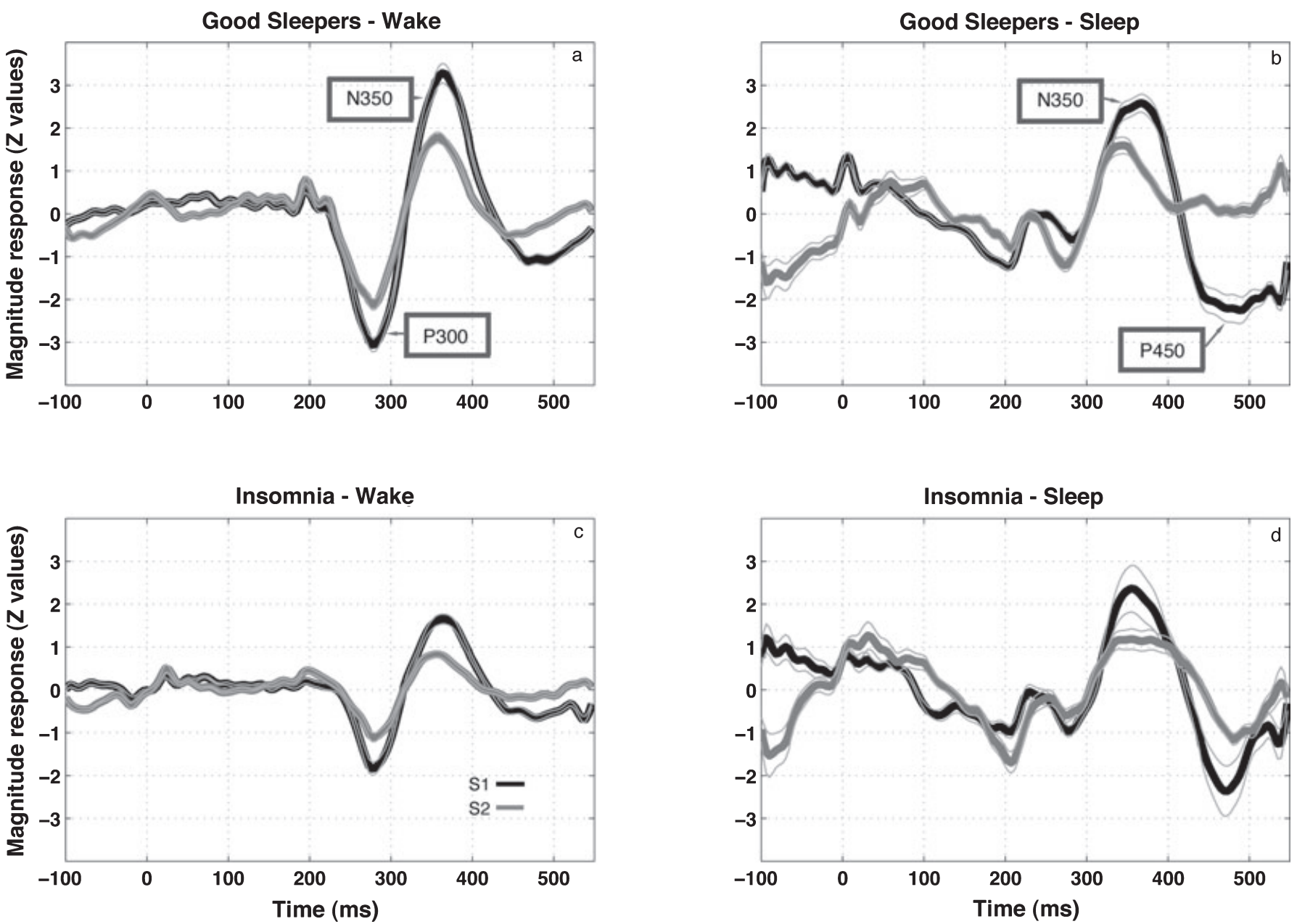

FIG. 2. ERPs evoked by stimulus presentation during baseline Wake (left panels) and Stage II sleep (right panels), for the two groups (controls, top panels; insomnia, lower panels). The darker thick line represents the ERP evoked by the presentation of S1, the lighter thick line the ERP evoked by S2. Thin light grey lines represent group standard error. During Wake (Fig. 1a and c) the evoked response consisted of two major components, a positive deflection around 300 ms (P300), and a negative deflection around $350 \mathrm{~ms}$ (N350). During sleep (Fig. 1b and d) P300 was diminished, N350 was still apparent and a positive deflection around 450 $500 \mathrm{~ms}$ (P450) appeared. 


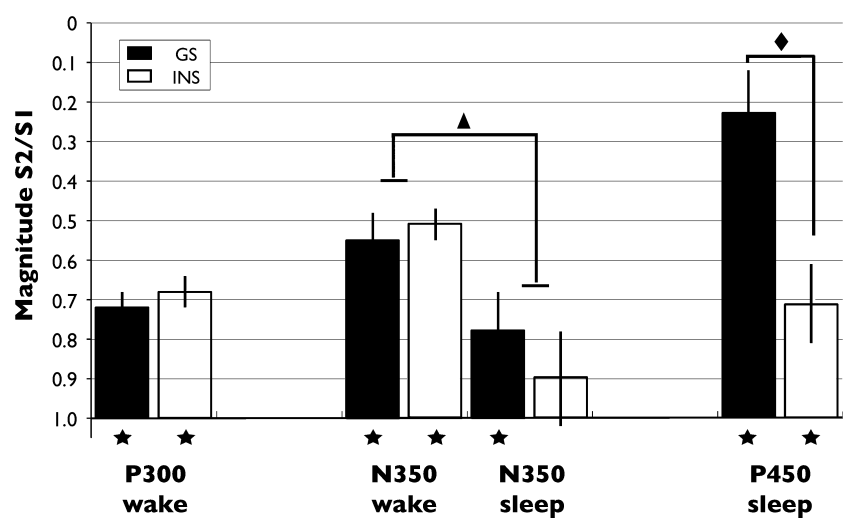

FIG. 3. Measures of gating of ERP components. Gating was assessed as the ratio of the second stimulus to the first $(\mathrm{S} 2 / \mathrm{S} 1)$. Note the ordinate axis is reversed. Stars represent statistically significant difference from a hypothesized ratio of 1 (no gating), based on independent sample $t$-tests. During wake, both groups (GS, dark bars; INS, white bars) showed significant gating of both ERP components, with no group differences. Groups did not differ in gating of P300 and waking N350; during sleep, gating of N350 was attenuated, and completely diminished in INS; gating of P450 was greater in GS. ' $\star$ ', gating significantly $<1$; ' $\boldsymbol{\Delta}$ ', difference between states; ' $\boldsymbol{}$ ', difference between groups.

but during sleep it was statistically $<1$ for GS $\left(t_{19}=-2.32\right.$, $P=0.034)$ but not for INS $\left(t_{17}=-1.27, P=0.22\right)$. Finally, univariate ANOVA on the GR of P450 yielded a main effect of group $\left(F_{1,35}=4.83, P=0.035, \eta_{\mathrm{p}}^{2}=0.13\right)$, due to greater gating in GS. One-sample $t$-tests revealed that the GRs were statistically $<1$ for both groups (GS: $t_{19}=-6.95, P<0.001$; INS: $t_{17}=-3.90, P=0.001$ ).

Figure $4 \mathrm{a}$ depicts the average number of KCs per epoch in Stage II. A repeated-measures ANOVA using the pre-stimulus baseline and during stimulus periods when KCs were counted yielded a significant interaction of group by period $\left(F_{1,41}=4.28, P=0.043, \eta_{\mathrm{p}}{ }^{2}=0.12\right)$, due to an increase in KCs during stimulus presentation in the GS group only. There was a trend towards a main effect of period $\left(F_{1,41}=2.73, P=0.108\right)$, and no main effect of group $(F<1.0)$. To determine whether the change in KCs was significant within subjects, the ratio of $\mathrm{KC}$ during stimulus presentation to the number of $\mathrm{KCs}$ in the $11 \mathrm{~min}$ prior to each block (pre-stimulus) was calculated, and one sample $t$-tests were run for each group, with a hypothesized mean of 1 (no change). As can be seen on the left-hand ordinate of Fig. 4a, there was almost a two-fold increase in GS $\left(t_{19}=3.44, P=0.001\right)$, with a non-significant change in INS participants $\left(t_{17}=1.27, P=0.205\right)$.

Equivalent analyses were conducted on the number of spindles counted during the same time periods (i.e. pre-stimulus, during stimulus; Fig. 4b). The INS group had significantly more spindles $\left(F_{1,41}=4.85, P=0.034, \quad \eta_{\mathrm{p}}{ }^{2}=0.11\right)$, with no effect of period $\left(F_{1,41}=0.74, P=0.395\right)$ or interaction $\left(F_{1,41}=0.096, P=0.758\right)$. The ratio analyses were also not significant for either group (GS: $t_{19}=1.31, P=0.101$, INS: $\left.t_{17}=1.37, P=0.089\right)$.

\section{Discussion}

As expected, participants with PI reported impaired subjective sleep quality (Table 2) corroborated by delayed objective SOL and lower sleep efficiency in the laboratory compared with GS participants (Table 3). Additionally, INS participants reported greater sleepiness in the morning, but not the evening (Table 2). Notably, INS participants had more depression and anxiety symptoms compared with GS (Table 2), but despite experiencing more mood difficulties INS did not differ from GS in income and education (Table 1), and did not meet criteria for other Axis I disorders.
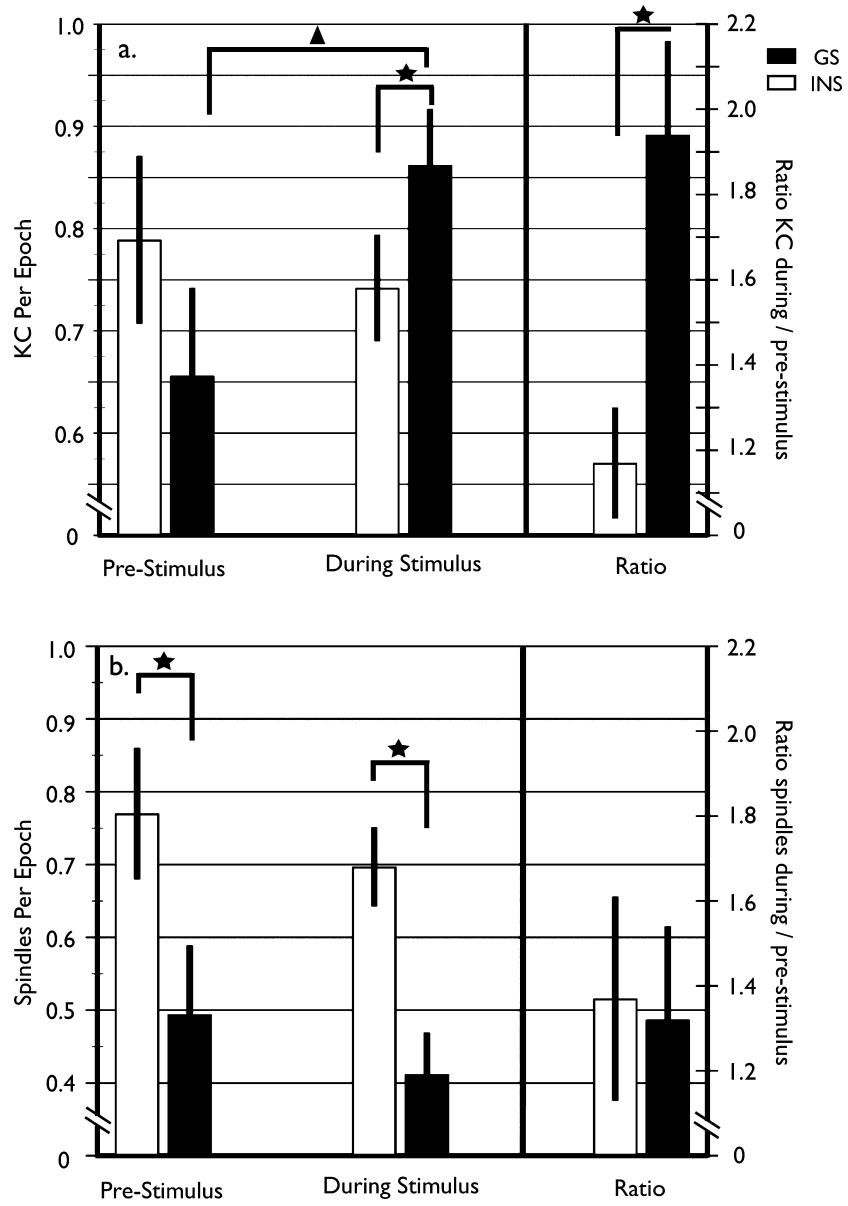

FIG. 4. KCs and spindles. KCs and spindles per epoch of Stage II/III were counted for the $11 \mathrm{~min}$ immediately preceding stimulus presentation, and during stimulus block. The ratio of these values was calculated and a $t$-test was run on this measure. (a) The left ordinate demonstrates that INS and GS did not differ in KCs in the absence of stimuli, while only GS showed a significant increase during stimulus presentation. The right ordinate demonstrates that GS almost doubles the number of KCs during stimulus presentation, while INS had no increase. (b) INS had significantly more spindles with no effect of stimulus presentation. ' $\star$ ' differences between groups; ' $\boldsymbol{\Delta}$ ' difference between before and during stimulus presentation.

Sleeping in the laboratory had differential effects on GS and INS participants, significantly reducing subjective sleep quality only for GS (Table 3). The introduction of the ERP stimulus did not further impair sleep quality in either group, and even improved sSOL for INS participants. This was corroborated by the fact that there was no increase in the number of recorded arousals when comparing acclimation to ERP night (Table 4). However, presentation of the ERP stimulus did facilitate transition out of SWS and REM into the lighter Stage II, with REMS being more stable in INS participants (Fig. 1).

We tested the hypothesis that PI is associated with an impaired mechanism for excluding irrelevant information during sleep using an ERP model of sensory gating. We used an ERP paradigm which has been shown to be sensitive to pre-attentive filtering of information to test our hypothesis. Auditory sensory gating is a neurophysiological phenomenon, typically observed when pairs of identical auditory stimuli are presented, resulting in an attenuation in the magnitude of ERP components induced by the second stimulus in the pair. It is assumed that the neural responses to the first stimulus activate an inhibitory gating mechanism that diminishes the response to the 
second one (Adler et al., 1998). Decreased gating, especially of the P50 ERP component, has been observed in several psychiatric disorders, including schizophrenia (Adler et al., 1998; Potter et al., 2006), and post-traumatic stress disorder (Neylan et al., 1999; Skinner et al., 1999). In addition, Kisley et al. (2001) demonstrated that in healthy participants P50 gating was largely maintained during sleep, and was even augmented during REM sleep. Although we did not observe mid-latency components (P50, N100) during either wake or sleep, our results generally support the hypothesis with distinct group differences in measures of gating during sleep. The waking ERP response consisted of two components, P300 and N350, and although INS participants had significantly attenuated response amplitude to both S1 and S2, both groups had clear gating of both components (Fig. 2).

P300 has been shown to be elicited both by attended salient stimuli and by stimuli that are irrelevant, with a slightly earlier positive deflection observed for the latter. Decreased amplitude and delayed latency of P300 has been reported in a range of mental and neurological disorders (Linden, 2005), including bipolar disorder (Fridberg et al., 2009) and agoraphobia (Gordeev, 2008), suggesting non-specific changes in neural organization required for eliciting this ERP component. Additionally, sleep deprivation and restriction may also reduce the amplitude of P300 (Edinger et al., 2004; Cote et al., 2008). Thus, the attenuation of the P300 during wake in PI participants is perhaps associated with the higher levels of anxiety and symptoms of depression.

During sleep, P300 is typically not observed, although there is some controversy regarding its occurrence during sleep (Cote, 2002), with reports of delayed onset and more frontal induction of this component (Bastuji \& García-Larrea, 1999; Colrain \& Campbell, 2007). In this study, ERP acquisition was done using $\mathrm{Cz}$, this may have contributed to the diminishment of this component during sleep.

N350 is reliably evoked by stimuli presented during sleep and sleep onset (Ogilvie et al., 1991; Hull \& Harsh, 2001), but has also been observed in response to interfering or competing stimuli while engaged in tasks that require high-level processing, such as decisionmaking or mental rotation (e.g. Schendan \& Kutas, 2003; Polezzi et al., 2008). Thus, it has been proposed that the N350 reflects a process that modulates (or gates) the perception of irrelevant stimuli (Harsh et al., 1994; Voss \& Harsh, 1998; Kallai et al., 2003), during both wake and sleep. Hence, the lower N350 amplitude observed in INS during wake may reflect reduced capacity to suppress processing of auditory stimuli while focusing on the film. Consistently, GS had significant gating during sleep, albeit attenuated compared with wake, whereas INS participants showed complete release from gating of N350 during sleep.

In contrast to mid-latency components, gating of N350 and P450 during sleep was maintained in GS. As noted above, gating of N350 was reduced relative to wake, especially in INS participants. Similarly, gating of P450 during sleep was significantly greater in GS participants. Bastuji \& García-Larrea (1999) suggested that N350 and $\mathrm{P} 450$ are early components of KCs. Indeed, Campbell et al. (1992) found that N350 amplitude is lower when it is not followed by a KC. Potentially, the 500-ms inter-stimulus interval was too short for the generation of two $\mathrm{KCs}$, resulting in a large gating ratio in the GS. Thus, sensory gating observed in GS, but to a lesser degree in INS, is perhaps secondary to the induction of the evoked $\mathrm{KC}$ that was attenuated in the INS group.

Similar to previous reports (Bastien et al., 2009b), the groups did not differ in the number of spontaneous KCs, although INS participants did not show an increase in the number of KCs during stimulus presentation. Both evoked and spontaneous KCs are assumed to be the manifestation of slow $(<1 \mathrm{~Hz})$ oscillations that occur during NREM sleep, which are the result of synchronous fluctuations in membrane potentials of cortical neurons (Amzica \& Steriade, 2002; Wilson et al., 2006). Moreover, the frequency of KCs increases closer to the onset of SWS, lending further support to the notion that the function of KCs is to facilitate slow-wave activity and to 'protect' the brain from interference (Bastien et al., 2000; Nicholas et al., 2002; Colrain, 2005). Thus, the observation that INS participants did not show an increase in KCs during stimulus presentation suggests that PI is associated with an impairment of mechanisms involved in protecting sleep from intrusive stimuli.

Additionally, INS participants had significantly more spindles than GS. Findings regarding spindle rate in PI are sparse and mixed with investigators reporting either no group differences between individuals with (psychophysiological) insomnia (Bastien et al., 2009a) or reduced spindling in individuals with sleep misperception (Besset et al., 1998). Further studies will help to elucidate whether PI is associated with differences in spindle activity. That said, spindle rate has been inversely associated with sleep pressure, with more spindles when sleep pressure has dissipated (Borbély et al., 1981; Finelli et al., 2001). This may suggest that sleep pressure dissipation is compromised in PI, which would be consistent with the elevated sleepiness reported by INS participants in their sleep diaries.

Although the findings described herein are largely convergent with the theoretical framework of the hyperarousal hypothesis, they should be interpreted with caution. First, it should be noted that in contrast to the typical auditory ERP (Näätänen, 1990) in this study P50 and N100 were not apparent in the averaged ERP response. This was potentially due to the short inter-trial interval compared with other studies (Ermutlu et al., 2007) and low sound intensity designed to minimize the probability of an arousal (Näätänen \& Picton, 1987). Indeed, the number of arousals recorded on the ERP night did not exceed that of the acclimation night (Table 2), information not typically provided in other sleep ERP studies. Similarly, during wake, visual attention attenuates the auditory N100 (Näätänen \& Picton, 1987; Oray et al., 2002). In this study, to make the waking state contextually more similar to sleep, participants watched a film with subtitles and were instructed to ignore the stimulus, which may have contributed to the suppression of the mid-latency components. Second, sleep studies conducted in the lab are inherently problematic as participants are not sleeping in their natural environment, which may also interact with the insomnia disorder. In fact, sleeping in the lab resulted in decreased subjective sleep quality on all measures for the GS but not INS participants. This was potentially due to the fact that the study was not conducted on consecutive nights, resulting in a 'first night effect' on both nights. Third, it should be noted that although we show a significant increase in the number of KCs during stimulus presentation, suggesting the existence of evoked $\mathrm{KCs}$, the parameters of the stimulus in the sensory gating ERP paradigm are not optimal to evoke KCs. Finally, due to an insufficient sample size and experimental design there were not enough ERP blocks from other sleep stages to address differences in sensory gating outside Stage II sleep. Expanding these finding to other sleep stages may help bolster our observations.

In summary, the findings reported provide preliminary evidence that sensory gating is impaired in PI during sleep, adding to a growing body of evidence that sleep difficulty in PI may be secondary to a fundamental disturbance of the central nervous system, resulting in altered processing and filtering of sensory information. The advantage of the sensory gating paradigm is that the neural mechanisms underlying both the ERP waveform and KCs have been studied 
extensively both in humans and in animal models, thus providing a foundation for understanding the neural mechanisms involved in PI and perhaps insomnia in general.

Importantly, despite evidence for group differences between individuals with insomnia and those without a sleep complaint on measures such as brain metabolism (Nofzinger et al., 2004) and highfrequency EEG activity (Perlis et al., 2001a,b), there are no objective measures for PI that can be assessed individually in a manner similar to apnoea (RDI) or periodic limb movement (PLM). Although further testing is necessary to determine the reliability of attenuated evoked $\mathrm{KCs}$ in individuals with PI, this measure is potentially a biomarker that can be readily adapted for clinical use to further characterize an individual's sleep difficulty.

\section{Acknowledgements}

Funding for this work was provided by NARSAD (A.G.H.), an APA postdoctoral fellowship (I.S.H.) and a UNCF-Merck postdoctoral fellowship (I.S.H.). We would like to thank the study participants who willingly subjected themselves to manipulations designed to interfere with their sleep and to all the dedicated research assistants who spent many nights awake. Special thanks go to Chris Knapp and Kerrie Hein who spent long hours on data analysis, to Irving Hafter and Ted Crum for help with sound intensity measurements, and to Jeff Kuznia from Compumedics, Brad Johnson and Noam Sobel for technical help with integrating ERP triggers into the Compumedics PSG system.

\section{Abbreviations}

(N)REM, (non-)rapid eye movement; BDI, Beck's depression inventory; ECG, electrocardiography; EEG, electroencephalography; EMG, electromyography; EOG, electrooculography; ERP, event-related potential; GR, gating ratio; GS, good sleepers; INS, insomnia; ISI, insomnia severity index; KC, K-complex; o, objective; PI, primary insomnia; PSG, polysomnography; s, subjective; SE, sleep efficiency; sNumW, subjective number of awakenings; SOL, sleep onset latency; SSS, Stanford sleepiness scale; STAI, state and trait anxiety inventory; SWS, slow-wave sleep; TST, total sleep time; WASO, wake after sleep onset.

\section{References}

Adler, L.E., Olincy, A., Waldo, M., Harris, J.G., Griffith, J., Stevens, K., Flach, K., Nagamoto, H., Bickford, P., Leonard, S. \& Freedman, R. (1998) Schizophrenia, sensory gating, and nicotinic receptors. Schizophr. Bull., 24, 189-202.

American Academy of Sleep Medicine. (2005) The International Classification of Sleep Disorders, Revised: Diagnostic and Coding Manual. American Academy of Sleep Medicine, Westchester, IL.

Amzica, F. \& Steriade, M. (2002) The functional significance of K-complexes. Sleep Med. Rev., 6, 139-149.

Bastien, C., Ladouceur, C. \& Campbell, K. (2000) EEG characteristics prior to and following the evoked K-complex. Canadian J. Exp. Psychol., 54, 255265.

Bastien, C.H., Vallieres, A. \& Morin, C.M. (2001) Validation of the Insomnia Severity Index as an outcome measure for insomnia research. Sleep Med, 2, 297-307.

Bastien, C.H., St-Jean, G., Morin, C.M., Turcotte, I. \& Carrier, J. (2008) Chronic psychophysiological insomnia: hyperarousal and/or inhibition deficits? An ERPs investigation Sleep, 31, 887-898.

Bastien, C.H., St-Jean, G., Turcotte, I., Morin, C.M., Lavallée, M. \& Carrier, J. (2009a) Sleep spindles in chronic psychophysiological insomnia. J. Psychosom. Res., 66, 59-65.

Bastien, C.H., St-Jean, G., Turcotte, I., Morin, C.M., Lavallee, M., Carrier, J. \& Forget, D. (2009b) Spontaneous K-complexes in chronic psychophysiological insomnia. J. Psychosom. Res., 67, 117-125.

Bastuji, H. \& García-Larrea, L. (1999) Evoked potentials as a tool for the investigation of human sleep. Sleep Med. Rev., 3, 23-45.

Beck, A.T., Steer, R.A. \& Brown, G.K. (1996) Manual for the Beck Depression Inventory-II. Psychological Corporation, San Antonio, TX.
Besset, A., Villemin, E., Tafti, M. \& Billiard, M. (1998) Homeostatic process and sleep spindles in patients with sleep-maintenance insomnia: effect of partial (21 h) sleep deprivation. Electroen. Clin. Neuro., 10, 122-132.

Bonnet, M.H. \& Arand, D.L. (1997) Hyperarousal and insomnia. Sleep Med. Rev., 1, 97-108.

Borbély, A.A., Baumann, F., Brandeis, D., Strauch, I. \& Lehmann, D. (1981) Sleep deprivation: effect on sleep stages and EEG power density in man. Electroencephalogr. Clin., 51, 483-493.

Buysse, D.J., Germain, A., Hall, M.L., Moul, D.E., Nofzinger, E.A., Begley, A., Ehlers, C.L., Thompson, W. \& Kupfer, D.J. (2008) EEG spectral analysis in primary insomnia: NREM period effects and sex differences. Sleep, 31, $1673-1682$.

Campbell, K.B., Bell, I. \& Bastien, C.H. (1992) Evoked potential measures of information processing during natural sleep. In Broughton, R.J. \& Ogilvie, R.D. (Eds), Sleep, Arousal, and Performance. Birkhauser, Boston, MA, pp. $88-116$.

Colrain, I.M. (2005) The K-complex: a 7-decade history. Sleep, 28, 255-273.

Colrain, I.M. \& Campbell, K.B. (2007) The use of evoked potentials in sleep research. Sleep Med. Rev., 11, 277-293.

Cote, K.A. (2002) Probing awareness during sleep with the auditory odd-ball paradigm. Int. J. Psychophysiol., 46, 227-241.

Cote, K.A., Milner, C.E., Osip, S.L., Baker, M.L. \& Cuthbert, B.P. (2008) Physiological arousal and attention during a week of continuous sleep restriction. Physiol. Behav., 95, 353-364.

Delorme, A. \& Makeig, S. (2004) EEGLAB: an open source toolbox for analysis of single-trial EEG dynamics including independent component analysis. J. Neurosci. Meth., 134, 9-21.

Edinger, J.D., Kirby, A.C., Lineberger, M.D., Loiselle, M.M., Wohlgemuth, W.K. \& Means, M.K. (2000) Duke Structured Interview Schedule for DSM$I V-T R$ and International Classification of Sleep Disorders, 2nd edn. Veterans Affairs and Duke University Medical Centers, Durham, North Carolina.

Edinger, J.D., Glenn, D.M., Bastian, L.A., Marsh, G.R., Daile, D., Hope, T.V., Young, M., Shaw, E. \& Meeks, G. (2001) Sleep in the laboratory and sleep at home II: comparisons of middle-aged insomnia sufferers and normal sleepers. Sleep, 24, 761-770.

Edinger, J.D., Bonnet, M.H., Bootzin, R.R., Doghramji, K., Dorsey, C.M., Espie, C.A., Jamieson, A.O., McCall, W.V., Morin, C.M. \& Stepanski, E.J. (2004) Derivation of research diagnostic criteria for insomnia: report of an American Academy of Sleep Medicine Work Group. Sleep, 27, 1567-1596.

Ermutlu, M.N.M.N., Demiralp, T.T. \& Karamürsel, S.S. (2007) The effects of interstimulus interval on sensory gating and on preattentive auditory memory in the oddball paradigm. Can magnitude of the sensory gating affect preattentive auditory comparison process?. Neurosci. Lett., 412, 1-5.

Espie, C.A. (2002) Insomnia: conceptual issues in the development, persistence, and treatment of sleep disorder in adults. Ann. Rev. Psychol., 53, 215243.

Finelli, L., Borbely, A. \& Acherman, P. (2001) Functional topography of the human non-REM sleep electroencephalogram. Eur. J. Neurosci., 13, 22822290 .

Fridberg, D.J., Hetrick, W.P., Brenner, C.A., Shekhar, A., Steffen, A.N., Malloy, F.W. \& O’Donnell, B.F. (2009) Relationships between auditory event-related potentials and mood state, medication, and comorbid psychiatric illness in patients with bipolar disorder. Bipolar. Disord., 11, 857-866.

Gordeev, S.A. (2008) Clinical-psychophysiological studies of patients with panic attacks with and without agoraphobic disorders. Neurosci. Behav. Physiol., 38, 633-637.

Harsh, J., Voss, U., Hull, J., Schrepfer, S. \& Badia, P. (1994) ERP and behavioral changes during the wake/sleep transition. Psychophysiology, 31, $244-252$.

Harvey, A.G. (2002) A cognitive model of insomnia. Behav. Res. Ther, 40, 869-893.

Hoddes, E., Zarcone, V., Smythe, H., Phillips, R. \& Dement, W.C. (1973) Quantification of sleepiness: a new approach. Psychophysiology, 10, 431436.

Hull, J. \& Harsh, J. (2001) P300 and sleep-related positive waveforms (P220, P450, and P900) have different determinants. J. Sleep Res., 10, 9-17.

Kallai, I., Harsh, J. \& Voss, U. (2003) Attention to external stimuli during wakefulness and sleep: evoked $40-\mathrm{Hz}$ response and N350. Psychophysiology, 40, 955-966.

Kisley, M.A., Olincy, A. \& Freedman, R. (2001) The effect of state on sensory gating: comparison of waking, REM and non-REM sleep. Clin. Neurophysiol., 112, 1154-1165.

Krystal, A.D., Edinger, J.D., Wohlgemuth, W.K. \& Marsh, G.R. (2002) NREM sleep EEG frequency spectral correlates of sleep complaints in primary insomnia subtypes. Sleep, 25, 630-640. 
Lamarche, C.H. \& Ogilvie, R.D. (1997) Electrophysiological changes during the sleep onset period of psychophysiological insomniacs, psychiatric insomniacs, and normal sleepers. Sleep, 20, 724-733.

Linden, D.E. (2005) The p300: where in the brain is it produced and what does it tell us? Neuroscientist, 11, 563-576.

Michael, B., Spitzer, R.L., Gibbon, M. \& Williams, J.B.W. (2002) Structured Clinical Interview for DSM-IV-TR Axis I Disorders, Research Version, Nonpatient Edition. (SCID-I/NP). New York: Biometrics Research, New York State Psychiatric Institute, New York.

Näätänen, R. (1990) The role of attention in auditory information processing as revealed by event-related potentials and other measures of cognitive function. Behav. Brain Sci., 13, 201-288.

Näätänen, R. \& Picton, T. (1987) The N1 wave of the human electric and magnetic response to sound: a review and an analysis of the component structure. Psychophysiology, 24, 375-425.

Neylan, T.C., Fletcher, D.J., Lenoci, M., McCallin, K., Weiss, D.S., Schoenfeld, F.B., Marmar, C.R. \& Fein, G. (1999) Sensory gating in chronic posttraumatic stress disorder: reduced auditory p50 suppression in combat veterans. Biol. Psychiatry, 46, 1656-1664.

Nicholas, C.L., Trinder, J. \& Colrain, I.M. (2002) Increased production of evoked and spontaneous K-complexes following a night of fragmented sleep. Sleep, 25, 882-887.

Nofzinger, E.A., Buysse, D.J., Germain, A., C., P.J., Miewald, J.M. \& Kupfer, D.J. (2004) Functional neuroimaging evidence for hyperarousal in insomnia. Am. J. Physiol., 161, 2126-2128.

Nofzinger, E.A., Nissen, C., Germain, A., Moul, D., Hall, M., Price, J.C., Miewald, J.M. \& Buysse, D.J. (2006) Regional cerebral metabolic correlates of WASO during NREM sleep in insomnia. J. Clin. Sleep Med., 2, 316-322.

Ogilvie, R.D., Simons, I.A., Kuderian, R.H., MacDonald, T. \& Rustenberg, J. (1991) Behavioral, event-related potential and EEG/FFT changes at sleep onset. Psychophysiology, 28, 54-64.

Oray, S., Lu, Z.L. \& Dawson, M.E. (2002) Modification of sudden onset auditory ERP by involuntary attention to visual stimuli. Int. J. Psychophysiol., 43, 213-224.

Patterson, J.V., Hetrick, W.P., Boutros, N.N., Jin, Y., Sandman, C., Stern, H., Potkin, S. \& Bunney, W.E. Jr (2008) P50 sensory gating ratios in schizophrenics and controls: a review and data analysis. Psychiatry Res., 158, 226-247.

Perlis, M.L., Giles, D.E., Mendelson, W.B., Bootzin, R.R. \& Wyatt, J.K. (1997) Psychophysiological insomnia: the behavioural model and a neurocognitive perspective. J. Sleep Res., 6, 179-188.
Perlis, M.L., Kehr, E.L., Smith, M.T., Andrews, P.J., Orff, H. \& Giles, D.E. (2001a) Temporal and stagewise distribution of high frequency EEG activity in patients with primary and secondary insomnia and in good sleeper controls. J. Sleep Res., 10, 93-104.

Perlis, M.L., Smith, M.T., Andrews, P.J., Orff, H. \& Giles, D.E. (2001b) Beta/Gamma EEG activity in patients with primary and secondary insomnia and good sleeper controls. Sleep, 24, 110-117.

Polezzi, D., Daum, I., Rubaltelli, E., Lotto, L., Civai, C., Sartori, G. \& Rumiati, R. (2008) Mentalizing in economic decision-making. Behav. Brain Res., 190, 218-223.

Potter, D., Summerfelt, A., J., G. \& Buchannan, R.W. (2006) Review of clinical correlates of P50 sensory gating abnormalities in patients with schizophrenia. Schizophr. Bull., 32, 692-700.

Rechtschaffen, A. \& Kales, A. (1968) A Manual of Standardised Terminology, Techniques and Scoring System for Sleep Stages of Human Subjects. Brain Information service/Brain Research Institute, Los Angeles.

Schendan, H.E. \& Kutas, M. (2003) Time course of processes and representations supporting visual object identification and memory. J. Cog. Neurosci., 15, $111-135$

Skinner, R.D., Rasco, L.M., Fitzgerald, J., Karson, C.N., Matthew, M., Williams, D.K. \& Garcia-Rill, E. (1999) Reduced sensory gating of the P1 potential in rape victims and combat veterans with posttraumatic stress disorder. Depress. Anxiety, 9, 122-130.

Spielberger, C.D., Gorscuch, R.L. \& Lushene, R.E. (1970) STAI Manual for the State-Trait Anxiety Inventory: ("Self-Evaluation Questionnaire"). Consulting Psychologists Press, Palo Alto, CA.

Spielman, A.J., Caruso, L.S. \& Glovinsky, P.B. (1987) A behavioral perspective on insomnia treatment. Psychiat. Clin. N. Am., 10, 541553.

Turcotte, I. \& Bastien, C.H. (2009) Is quality of sleep related to the N1 and P2 ERPs in chronic psychophysiological insomnia sufferers? Int. J. Psychophysiol., 72, 314-322.

Voss, U. \& Harsh, J. (1998) Information processing and coping style during the wake/sleep transition. J. Sleep Res., 7, 225-232.

Wilson, M.T., Steyn-Ross, D.A., Sleigh, J.W., Steyn-Ross, M.L., Wilcocks, L.C. \& Gillies, I.P. (2006) The K-complex and slow oscillation in terms of a mean-field cortical model. J. Comput. Neurosci., 21, 243-257.

Yang, C.-M. \& Lo, H.-S. (2007) ERP evidence of enhanced excitatory and reduced inhibitory processes of auditory stimuli during sleep in patients with primary insomnia. Sleep, 30, 585-592. 